\title{
PERFIL EPIDEMIOLÓGICO DA CÁRIE DENTÁRIA, CONDIÇÕES PERIODONTAIS E HIGIENE ORAL EM ESCOLARES DO MUNICÍPIO DE CAAPORÃ - PB, BRASIL
}

\section{EPIDEMIOLOGICAL PROFILE OF DENTAL CARIES, PERIODONTAL CONDITIONS AND ORAL HYGIENE IN STUDENTS OF CAAPORÃ-PB, BRAZIL}

\author{
Mariana Leonel Martins*, George Max de Oliveira Cartaxo**, Wilton Wilney Nascimento \\ Padilha***
}

Autor para correspondência: Wilton Wilney Nascimento Padilha - wiltonpadilha@yahoo.com.br

*Graduanda do Curso de Odontologia da Universidade Federal da Paraíba, João Pessoa-PB, Brasil.

**Cirurgião Dentista do Centro de Especialidades Odontológicas do município de Cajazeiras-PB.

***Professor Titular do Departamento de Clínica e Odontologia Social da Universidade Federal da Paraíba, João Pessoa-PB, Brasil.

OBJETIVO: Analisar as condições de saúde bucal de escolares de Caaporã-PB quanto à prevalência de cárie, alterações periodontais e qualidade de higiene oral. METODOLOGIA: Realizou-se um estudo transversal com 320 alunos distribuídos nas idades de 5, 12 e 15 a 19 anos com 62, 112 e 146 escolares, respectivamente. O nível de confiança foi $95 \%$, com $5 \%$ de margem de erro e amostragem selecionada de modo não aleatório. Utilizou-se os índices ceo-d e CPO-D (dentes cariados, perdidos e obturados), o Índice de Higiene Oral Simplificado (IHOS) e o Índice Periodontal Comunitário (IPC) para avaliar sangramento gengival e cálculo dentário. Efetuou-se análise descritiva e inferencial dos dados com teste Qui-quadrado $(\alpha=0,05)$. RESULTADOS: $O$ ceo- $d$ foi $3,69 \pm 4,71$ aos 5 anos e $42,0 \%$ não tiveram experiência de cárie. O CPO-D obtido aos 12 e 15 a 19 anos foi, respectivamente, 2,44 2,60

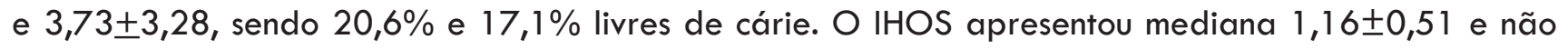
houve diferença em relação à cárie $(p>0,05)$. Verificou-se que $90,1 \%$ dos escolares que apresentaram sangramento gengival e $85,7 \%$ dos que apresentaram cálculo tinham $C P O \geq 1 \quad(p<0,05)$. CONCLUSÕES: A prevalência da cárie foi considerada alta aos 5 anos, baixa aos 12 e média dos 15 aos 19 anos. A higiene oral foi classificada como regular, sendo mais deficiente nas crianças de 5 anos. Houve associação $(p<0,05)$ entre cárie e condições de sangramento gengival $(R P=3,117 ; I C=1,332-7,296)$ e cálculo dentário ( $R P=1,983 ; I C=1,027-3,829)$. Esses resultados possibilitam o direcionamento das ações em saúde e, consequentemente, a obtenção de melhores condições bucais.

PALAVRAS-CHAVE: Cárie Dentária; Inquéritos Epidemiológicos; Levantamentos de Saúde Bucal. 
OBJECTIVE: Analyze the oral health conditions of Caaporã-PB students in the dental caries prevalence, periodontal changes and quality of oral hygiene. MATERIAL AND METHOD: A cross-sectional study with 320 students in the ages of 5,12 and 15 to 19 years with 62, 112 and 146 students, respectively was conducted. The confidence level was $95 \%$, with a $5 \%$ margin of error and the sample wasn't randomly selected. The dmft and DMFT indices for decayed, missing and filled teeth, the Simplified Oral Hygiene Index (SOHI), and Community Periodontal Index (CPI) to assess bleeding gingival and teeth calculus were employed. A descriptive and inferential analysis of the data with Chi-square test $(\alpha=0.05)$ was conducted. RESULTS: The dmft was $3.69 \pm 4.71$ at 5 years and $42 \%$ hadn't caries experience. The DMFT was obtained, respectively, $2.44 \pm 2.60$ and $3.73 \pm 3.28$ at 12 and 15 to 19 years, with $20.6 \%$ and $17.1 \%$ without caries experience. The median of $\mathrm{SOHI}$ was $1.16 \pm 0.51$ and there wasn't difference in relation to caries $(p>0.05)$. It was found that $90.1 \%$ of students with gum bleeding and $85.7 \%$ with teeth calculus had DMFT $\geq 1 \quad(p<0.05)$. CONCLUSION: The caries prevalence was considered high at 5 years, low to 12 and mean at 15 to 19 years. Oral hygiene was classified as regular, being more deficient in children of 5 years. There was association $(p<0.05)$ between decay and gum bleeding conditions $(\mathrm{OR}=3.117, \mathrm{Cl}=1.332-7.296)$ and dental calculus $(\mathrm{OR}=1.983, \mathrm{Cl}=1.027-3.829)$. These results allow the directing of health actions and therefore obtain better oral conditions.

KEYWORDS: Dental Caries; Health Surveys; Dental Health Surveys. 


\section{INTRODUÇÃO}

O acompanhamento das condições em saúde bucal por um longo período de tempo através de levantamentos epidemiológicos possibilita a avaliação das intervenções realizadas e sua efetividade, colaborando para o planejamento e desenvolvimento de iniciativas em saúde'.

O mais recente levantamento epidemiológico de saúde bucal de base nacional foi o SB Brasil 2010, no qual participaram indivíduos residentes em 177 municípios das cinco macrorregiões. Foram realizados exames bucais para avaliar as condições de saúde e os principais agravos e utilizou-se de questionários para coleta de dados sobre a condição socioeconômica, acesso aos serviços odontológicos e percepção de saúde ${ }^{2}$.

A verificação da atividade de cárie por meio de levantamentos epidemiológicos permite - diagnóstico precoce de lesões tornando o tratamento mais simples, menos invasivo e com menor custo, envolvendo uso de flúor e mudanças de comportamento com relação à dieta e à higiene oral. Dessa forma, os resultados encontrados a partir desses levantamentos podem ser utilizados para direcionar os serviços odontológicos para essa população, interrompendo a evolução natural da cárie $^{3}$.

Conforme os resultados apresentados no SB Brasil 2010, o número de indivíduos livres de cárie (ceo/ $\mathrm{CPO}=0$ ) diminui em função da idade, provavelmente devido ao número crescente de dentes erupcionados e ao caráter cumulativo dos índices utilizados. Aos 5 anos de idade, $46,6 \%$ das crianças brasileiras estão livres de cárie na dentição decídua e aos 12 anos, $43,5 \%$ apresentam esta condição na dentição permanente. Na faixa etária de 15 a 19 anos esse percentual é de $23,9 \%{ }^{2}$.

As macrorregiões brasileiras apresentam perfis divergentes para cada idade, sendo as regiões Sul e Sudeste as que possuem maiores proporções de indivíduos livres de cárie. Quanto ao Nordeste e considerando a faixa etária de 5 anos, Maceió foi a capital que apresentou maior valor do ceo-d $(2,76)$, porém esta foi inferior a média dos municípios do interior da região $(3,94)$. Nas faixas etárias de 12 e 15 a 19 anos, João Pessoa foi a capital com maior CPO-D (2,78 e 6,15, respectivamente), entretanto esses valores foram menores que os observados nos municípios do interior do Nordeste (CPO-D=3,84 e 6,22 , respectivamente). Portanto, podemos afirmar que a experiência de cárie na região Nordeste é maior nos municípios localizados no interior do que nas capitais ${ }^{2}$.

A prevenção da cárie dentária na primeira infância requer ações amplas e multidisciplinares, visto que a etiologia desta doença é complexa e que crianças com lesões de cárie na primeira dentição estão mais predispostas a desenvolvê-las também na dentadura mista e permanente ${ }^{4}$. $E$, para obter uma melhor oclusão, é necessária a permanência dos dentes decíduos até o período de erupção dos permanentes, de forma a contribuir para $\circ$ posicionamento correto dos dentes permanentes ${ }^{5}$.

As alterações periodontais, assim como a cárie dentária, podem ter sua principal etiologia associada ao acúmulo de biofilme dentário, decorrente da má higienização bucal ${ }^{6}$. Segundo o SB Brasil 2010, as periodontopatias acometem 37,1\% das crianças brasileiras de 12 anos de idade, sendo - maior percentual encontrado na região Norte $(58,4 \%)$ e o menor na região Sudeste $(32,1 \%)$. Dentre os adolescentes de 15 a 19 anos, $49,1 \%$ apresentaram alguma alteração periodontal, sendo o cálculo dentário a alteração mais presente $(28,4 \%)$. A região Norte também apresentou os piores resultados para esta faixa etária, na qual $69,2 \%$ dos examinados tiveram alguma alteração periodontal, enquanto que na região Sudeste $(43,2 \%)$ foram encontradas as melhores condições ${ }^{2}$.

O presente estudo foi realizado no município de Caaporã, localizado na região Metropolitana de João Pessoa. A rede de saúde deste município é formada por 9 equipes de Saúde da Família, 9 equipes de Saúde Bucal e 3 cirurgiões-dentistas no centro de especialidades odontológicas. Atualmente a Prefeitura de Caaporã desenvolve ações de saúde bucal articulando a rede de escolas e a rede de saúde, conforme propõe o Programa Saúde na Escola ${ }^{7}$. Estas ações constituem-se de palestras, escovação supervisionada e aplicação tópica de fúor, com ênfase na higiene e prevenção da cárie dentária, porém não estão sistematizadas para controle de cobertura ou de eficácia. As demais 
ações em saúde bucal para escolares funcionam em sistema de livre demanda.

Portanto, essa pesquisa teve como objetivo analisar as condições de saúde bucal de escolares de 5, 12 e 15 a 19 anos do município de Caaporã em relação à prevalência da cárie dentária, às alterações periodontais e à qualidade de higiene oral, nos anos de 2013 e 2014.

\section{METODOLOGIA}

\section{Delineamento geral do estudo}

Realizou-se um estudo epidemiológico transversal com uma abordagem indutiva, procedimento comparativo e estatístico e técnica de documentação direta intensiva por meio de fichas clínicas ${ }^{8}$, utilizando - procedimento preconizado pela Organização Mundial de Saúde $(\mathrm{OMS})^{9}$ para a realização de estudos epidemiológicos e foram adotados os códigos e critérios do Projeto SB Brasil $2010^{2}$.

Esta pesquisa foi desenvolvida no município de Caaporã, localizado a $60 \mathrm{~km}$ da cidade de João Pessoa, capital da Paraíba, com 20.653 habitantes distribuídos em uma área de $150 \mathrm{~km}^{2}$ e Índice de Desenvolvimento Humano (IDH) igual a 0,6010,1 .

\section{Considerações éticas}

Esta pesquisa foi aprovada pelo Comitê de Ética em Pesquisa do Centro de Ciências da Saúde da Universidade Federal da Paraíba, CAAE 18184913.0 .0000 .5188 . Os alunos com idades de 5,12 e 15 a 19 anos foram convidados a participar e, após receberem informações sobre a pesquisa e concordarem em participar da mesma, assinando o Termo de Assentimento do Menor (TAM) - exceto as crianças de 5 anos, e, o responsável, o Termo de Consentimento Livre e Esclarecido (TCLE), foram examinados.

\section{Universo e amostra}

Fizeram parte da pesquisa alunos de duas escolas públicas do município de Caaporã-PB. $O$ universo foi composto por crianças e adolescentes do ensino fundamental das 21 escolas e creches municipais de Caaporã-PB, sendo destas 3 escolas públicas da zona rural, e a amostra foi definida mediante cálculo amostral.

O tamanho da amostra teve $95 \%$ de confiança e 5\% de margem de erro conforme número de estudantes das duas maiores escolas municipais de Caaporã, onde foi realizada esta pesquisa. As escolas foram selecionadas de modo não aleatório, devido ao seu porte e facilidade de acesso.

\section{Calibração da equipe envolvida}

A calibração da equipe foi realizada em duas etapas, uma teórica e outra prática. Esta última foi realizada por meio de exames clínicos de estudantes de 5 anos, em uma creche, e de 12 e 15 a 19 anos em uma escola, ambas do município de João Pessoa-PB, com duração de 18 horas. Obteve-se coeficiente kappa inter-examinador de 0,77 para cárie dentária, 0,87 para higiene oral e 0,66 para condição periodontal.

\section{Coleta de dados}

Os dados foram coletados semanalmente, de novembro de 2013 a julho de 2014, por uma equipe de pesquisadores composta por dois examinadores e dois anotadores. Um dos examinadores estava na condição de cirurgião-dentista (G.M.O.C.) e o outro como acadêmico do curso de Odontologia (M.L.M.); enquanto que os dois anotadores estavam na condição de acadêmicos do curso de Odontologia.

Os índices ceo-d e CPO-D foram utilizados para avaliar, respectivamente, dentes decíduos e permanentes, cariados, com extração indicada/ perdidose obturados 9 . A classificação da prevalência de cárie empregou critérios estabelecidos pela OMS (1982), baseada na média do índice CPO-D. Utilizouse a faixa etária de 12 anos como referência por ser considerada idade-índice e a mais utilizada nos levantamentos epidemiológicos. Esta classificação considera a prevalência como muito baixa $(0,0$ a $1,1)$, baixa $(1,2$ a 2,6$)$, média $(2,7$ a 4,4$)$, alta $(4,5$ a 6,5$)$ ou muito alta $(\geq 6,6)^{12}$. No presente estudo foi realizada uma adaptação desta classificação para cada faixa etária, considerando a evolução da saúde bucal e o atual perfil da cárie.

O Índice de Higiene Oral Simplificado (IHOS) objetivou mensurar a qualidade de higiene a partir da presença de biofilme nos dentes índices $(16,11$, $26,31,36$ e 46 ), representantes dos elementos 
anteriores e posteriores da arcada dentária. A higiene oral foi categorizada em: boa $(0,0-0,6)$, regular $(0,7-1,8)$ ou ruim $(1,9-3,0)^{13}$.

A presença de sangramento gengival após estímulo e cálculo dentário em escolares de 12 e 15 a 19 anos foi avaliada por meio do Índice Periodontal Comunitário (IPC), que considera os mesmos dentes índices do $\operatorname{IHOS}(16,11,26,31,36$ e 46) 9

\section{Instrumentos utilizados}

O exame clínico foi realizado na biblioteca das escolas, buscando o máximo de aproveitamento da luz natural, utilizando-se a sonda WHO,

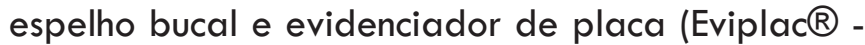
Biodinâmica, Rio de Janeiro-RJ, Brasil), e os dados foram registrados em ficha clínica, adaptada do SB Brasil 2010², elaborada para a pesquisa, adotando os códigos e critérios preconizados pela $\mathrm{OMS}^{9}$.

Análise dos dados

Os dados coletados foram transferidos para 0 banco de dados elaborado no software SPSS 17.0 (Inc, Chicago, IL, USA) e efetuou-se análise descritiva e inferencial com teste Qui-quadrado $(\alpha=0,05)$.

\section{RESULTADOS}

Fizeram parte desse estudo 320 alunos com idades de $5(n=62), 12(n=112)$ e 15 a 19 anos $(n=146)$. A coleta de dados foi realizada nas Escolas Severina Helena ( $n=217)$ e Adauto Viana ( $=103)$, ambas localizadas no município de Caaporã, onde foram realizados os exames clínicos.

A prevalência de cárie dentária neste estudo foi de 76,9\% ( $n=246)$, sendo considerada alta aos 5 anos, idade na qual era esperada uma menor exposição aos fatores etiológicos da cárie do que os outros grupos devido a baixa idade. Aos 12 e 15 a 19 anos, a prevalência de cárie foi considerada, respectivamente, baixa e média. Vale ressaltar que tais parâmetros foram estabelecidos para serem alcançados até o ano 2000, sendo atualmente necessário considerar a redução da cárie, bem como a polarização da mesma, a nível mundial (Tabela 1).

Houve um aumento na prevalência de cárie com - avançar da idade, resultado este já esperado devido ao caráter cumulativo do índice. A cárie acometeu com maior frequência o sexo feminino $(52,0 \%)$, porém sem diferença estatisticamente significativa (Tabela 1).

\begin{tabular}{|c|c|c|c|c|c|c|}
\hline \multirow[b]{2}{*}{ Idade } & \multicolumn{2}{|c|}{ Experiência de cárie } & \multirow[b]{2}{*}{$\begin{array}{c}\text { Grupo total } \\
\mathrm{n}(\%)\end{array}$} & \multirow[b]{2}{*}{ Média } & \multirow{2}{*}{\multicolumn{2}{|c|}{ Prevalência }} \\
\hline & $\begin{array}{c}\text { Sim } \\
n(\%)\end{array}$ & $\begin{array}{l}\text { Não } \\
\text { n (\%) }\end{array}$ & & & & \\
\hline 5 anos & $36(58,0)$ & $26(42,0)$ & $62(100)$ & 3,69 & & Alta \\
\hline 12 anos & $89(79,4)$ & $23(20,6)$ & $112(100)$ & 2,45 & & Baixa \\
\hline 15 a 19 anos & $121(82,9)$ & $25(17,1)$ & $146(100)$ & 3,73 & & Média \\
\hline Total & $246(76,9)$ & $74(23,1)$ & $320(100)$ & - & & - \\
\hline Variável & $\begin{array}{c}\text { Experiên } \\
\text { Sim } \\
\text { n (\%) }\end{array}$ & $\begin{array}{c}\text { de cárie } \\
\text { Não } \\
\text { n (\%) }\end{array}$ & $\begin{array}{c}\text { Grupo total } \\
n(\%)\end{array}$ & Valor de p & $\mathbf{R P}$ & IC \\
\hline \multicolumn{7}{|l|}{ Sexo } \\
\hline Feminino & $128(79,0)$ & $34(21,0)$ & $162(100)$ & & & \\
\hline Masculino & $118(74,7)$ & $40(25,3)$ & $158(100)$ & $\mathrm{p}>0,05$ & 1,276 & $0,758-2,149$ \\
\hline Total & $246(76,9)$ & $74(23,1)$ & $320(100)$ & & & \\
\hline
\end{tabular}

*Teste Qui-quadrado $(\mathrm{p}<0,05)$. 
O número de dentes decíduos hígidos e de elementos dentários componentes do ceo-d foi de, respectivamente, $966(80 \%)$ e $242(20 \%)$, enquanto que para a dentição permanente foi de 6337 $(88,5 \%)$ hígidos e 821 (11,5\%) dentes compondo o CPO-D (Tabela 2).
O componente cariado representou $78,1 \%$ do ceo-d e $47,1 \%$ do CPO-D. O número de restaurações sem recidiva de cárie na dentição permanente $(37,3 \%)$ correspondeu a um percentual superior ao da dentição decídua $(9,1 \%)$ (Tabela 2 ).

\begin{tabular}{|c|c|c|}
\hline Dentes decíduos $(\mathrm{n}=1208)$ & $\mathrm{n}$ & $\%$ \\
\hline Hígidos & 966 & 80 \\
\hline Com experiência de cárie & 242 & 20 \\
\hline Total & 1208 & 100 \\
\hline Componentes do ceo-d $(n=242)$ & $\mathrm{n}$ & $\%$ \\
\hline Cariados & 189 & 78,1 \\
\hline Extração indicada & 31 & 12,8 \\
\hline Obturados & 22 & 9,1 \\
\hline Total & 242 & 100 \\
\hline Dentes permanentes $(n=7158)$ & $\mathrm{n}$ & $\%$ \\
\hline Hígidos & 6337 & 88,5 \\
\hline Com experiência de cárie & 821 & 11,5 \\
\hline Total & 7158 & 100 \\
\hline Componentes do CPO-D $(n=821)$ & $\mathrm{n}$ & $\%$ \\
\hline Cariados & 387 & 47,1 \\
\hline Perdidos & 128 & 15,6 \\
\hline Obturados & 306 & 37,3 \\
\hline Total & 821 & 100 \\
\hline
\end{tabular}

A higiene oral foi considerada boa, regular e ruim em, respectivamente, $23,5 \%(n=75), 67,2 \%$ $(n=215)$ e $5,3 \%(n=17)$ da população estudada. Este índice não foi avaliado em 4,0\% ( $n=13)$ da população estudada. Para a realização da análise estatística, as condições de higiene regular e ruim foram agrupadas. A qualidade da higiene oral não foi associada à experiência de cárie ( $p>0,05$; $R P=1,289 ; I C=0,709-2,346$ ) (Tabela 3). Observouse que as crianças de 5 anos, cuja mediana foi $1,25$ ( $\pm 0,65)$, apresentaram pior higiene quando comparadas aos indivíduos de 12 e de 15 a 19 anos, que tiveram mediana igual a 1,0 , e desvio padrão de 0,45 e 0,52, respectivamente.

\begin{tabular}{|c|c|c|c|c|c|c|}
\hline \multirow[b]{2}{*}{ Variável } & \multicolumn{2}{|c|}{ Experiência de cárie } & \multirow[b]{2}{*}{$\begin{array}{c}\text { Grupo total } \\
\text { n (\%) }\end{array}$} & \multirow[b]{2}{*}{ Valor de p } & \multirow[b]{2}{*}{$\mathbf{R P}$} & \multirow[b]{2}{*}{ IC } \\
\hline & $\begin{array}{l}\text { Sim } \\
\mathrm{n}(\%)\end{array}$ & $\begin{array}{l}\text { Não } \\
\text { n(\%) }\end{array}$ & & & & \\
\hline \multicolumn{7}{|c|}{ Higiene oral } \\
\hline Boa & $54(72,0)$ & $21(28,0)$ & $75(100)$ & & & \\
\hline Regular** & $181(78,0)$ & $51(22,0)$ & $232(100)$ & $\mathrm{p}>0,05$ & 1,289 & $0,709-2,346$ \\
\hline Total & $235(76,5)$ & $72(23,5)$ & $307(100)$ & & & \\
\hline
\end{tabular}


Verificou-se que $\circ$ sangramento gengival esteve presente em $28,6 \%$ da população estudada e não foi associado à idade ( $>00,05 ; R P=1,226$; $\mathrm{IC}=0,698-2,156)$. O cálculo dentário, porém, foi observado em $40,7 \%$ dos estudantes, acometendo com maior frequência $(65,7 \%)$ os adolescentes de 15 a 19 anos $(p<0,05 ; R P=1,892 ; I C=1,133-3,159)$
(Tabela 4). Na tabela 5, observa-se que houve diferença estatisticamente significativa $(p<0,05)$ entre experiência de cárie e condição periodontal, seja considerando a presença de sangramento gengival $(R P=3,117 ; \quad I C=1,332-7,296)$ ou do cálculo dentário $(\mathrm{RP}=1,983 ; \mathrm{IC}=1,027-3,829)$.

\begin{tabular}{|c|c|c|c|c|c|c|}
\hline \multirow[b]{2}{*}{ Variável } & \multicolumn{2}{|c|}{ Sangramento } & \multirow[b]{2}{*}{$\begin{array}{c}\text { Grupo total } \\
\mathrm{n}(\%)\end{array}$} & \multirow[b]{2}{*}{ Valor de $p$} & \multirow[b]{2}{*}{$\mathbf{R P}$} & \multirow[b]{2}{*}{ IC } \\
\hline & $\begin{array}{l}\text { Sim } \\
n(\%)\end{array}$ & $\begin{array}{l}\text { Não } \\
\text { n (\%) }\end{array}$ & & & & \\
\hline \multicolumn{7}{|l|}{ Idade } \\
\hline 12 anos & $27(26,2)$ & $76(73,8)$ & $103(100)$ & & & \\
\hline 15 a 19 anos & $44(30,3)$ & $101(69,7)$ & $145(100)$ & $p>0,05$ & 1,226 & $0,698-2,156$ \\
\hline Total & $71(28,6)$ & $177(71,4)$ & $248(100)$ & & & \\
\hline Variável & $\begin{array}{l}\text { Cálculc } \\
\text { Sim } \\
\text { n (\%) }\end{array}$ & $\begin{array}{c}\text { entário } \\
\text { Não } \\
\text { n (\%) }\end{array}$ & $\begin{array}{c}\text { Grupo total } \\
\mathrm{n}(\%)\end{array}$ & Valor de $p$ & $\mathbf{R P}$ & IC \\
\hline \multicolumn{7}{|l|}{ Idade } \\
\hline 12 anos & $36(32,1)$ & $76(67,9)$ & $112(100)$ & & & \\
\hline 15 a 19 anos & $69(47,3)$ & $77(52,7)$ & $146(100)$ & $\mathrm{p}<0,05$ & 1,892 & $1,133-3,159$ \\
\hline Total & $105(40,7)$ & $153(59,3)$ & $258(100)$ & & & \\
\hline
\end{tabular}

*Teste Qui-quadrado $(\mathrm{p}<0,05)$.

\begin{tabular}{|c|c|c|c|c|c|c|}
\hline \multirow[b]{2}{*}{ Variável } & \multicolumn{2}{|c|}{ Experiência de cárie } & \multirow[b]{2}{*}{$\begin{array}{c}\text { Grupo total } \\
\text { n (\%) }\end{array}$} & \multirow[b]{2}{*}{ Valor de $p$} & \multirow[b]{2}{*}{$\mathbf{R P}$} & \multirow[b]{2}{*}{ IC } \\
\hline & $\begin{array}{c}\mathrm{CPO} \geq \mathbf{1} \\
\text { n }(\%)\end{array}$ & $\begin{array}{c}\mathrm{CPO}=0 \\
\mathrm{n}(\%)\end{array}$ & & & & \\
\hline \multicolumn{7}{|c|}{ Sangramento } \\
\hline $\operatorname{Sim}$ & $64(90,1)$ & $7(9,9)$ & $71(100)$ & & & \\
\hline Não & $132(74,6)$ & $45(25,4)$ & $177(100)$ & $\mathrm{p}<0,05$ & 3,117 & $1,332-7,296$ \\
\hline Total & $196(79,0)$ & $52(21,0)$ & $248(100)$ & & & \\
\hline \multicolumn{7}{|l|}{ Cálculo } \\
\hline $\operatorname{Sim}$ & $90(85,7)$ & $15(14,3)$ & $105(100)$ & & & \\
\hline Não & $115(75,2)$ & $38(24,8)$ & $153(100)$ & $\mathrm{p}<0,05$ & 1,983 & $1,027-3,829$ \\
\hline Total & $205(79,5)$ & $53(20,5)$ & $258(100)$ & & & \\
\hline
\end{tabular}

\section{DISCUSSÃO}

Osindivíduos comexperiência de cárie representaram a maior parte da população estudada $(76,9 \%)$. Um estudo indicou que cidades das regiões Nordeste e Centro-Oeste representam 50,6\% do grupo com maior severidade de cárie dentária, comprovando que a experiência de cárie no Brasil é influenciada por diversidades geográficas ${ }^{14}$.

A redução da cárie pode estar associada a 
diversos fatores, como a fluoretação das águas de abastecimento público, o maior acesso aos serviços odontológicos e às ações coletivas de saúde, melhor higiene oral e educação em saúde $\mathrm{e}^{3,15-18}$. A água de abastecimento público do município de Caaporã não recebe adição de flúor na estação de tratamento e, o desenvolvimento de ações de promoção de saúde, como a distribuição de escovas de dente e dentifrícios fluoretados, não garante uma melhoria nas condições de saúde bucal.

Os resultados obtidos com relação ao sexo dos alunos estudados foram trabalhados em conjunto, visto que não apresentaram diferença estatística significativa ${ }^{18}$, assim como observado em escolares do município de Carapicuíba-SP, no qual a experiência de cárie do gênero feminino correspondeu a $51,8 \%$, enquanto que no presente estudo $\circ$ percentual foi de $52 \%{ }^{19}$. Resultado semelhante também foi observado em escolares de Campina Grande-PB ${ }^{20}$, Recife-PE e Feira de Santana-BA ${ }^{21}$.

O Índice de Desenvolvimento Humano (IDH) da cidade de Caaporã $(0,60), 68^{\circ}$ no ranking da Paraíba, é inferior ao da capital João Pessoa $(0,76)$, que é $\circ 1^{\circ}$ no ranking do estado, e menor que a média do Brasil $(0,73)^{10}$. O menor desenvolvimento pode contribuir para o agravamento das condições de cárie na medida em que significam menor acesso aos serviços odontológicos e aos produtos de higiene bucal. Esta hipótese fundamenta o resultado encontrado, que foi um pior CPO-D aos 12 anos em Caaporã $(2,44)$ quando comparado à média nacional $(2,07)^{2}$. Entretanto, em um levantamento epidemiológico realizado no Macapá/AP observouse que, apesar do IDH do estado $(0,78)$ ser superior à média nacional, foi encontrada uma experiência de cárie mais alta nas crianças macapaenses do que a média das crianças brasileiras ${ }^{18}$.

O ceo-d dos estudantes de Caaporã aos 5 anos $(3,69)$ foi mais elevado que em Bayeux $(2,86)$, município também localizado na Região Metropolitana de João Pessoa. Porém, dentre os estudantes de Bayeux apenas $32,1 \%$ apresentavam ceo- $d=0$, enquanto que em Caaporã esse número foi de $42,0 \%$. Deste modo, a condição encontrada em Caaporã foi a que mais se aproximou do proposto pela OMS para o ano de 2000 (50\% livres de cárie), sendo esse parâmetro um dos mais importantes indicadores de saúde bucal ${ }^{16}$. Este resultado sugere que as crianças de Caaporã que apresentaram experiência de cárie tiveram um maior número de dentes acometidos pela doença do que as crianças de Bayeux, o que elevou a média do ceo-d. Apesar da localização próxima entre os municípios, Caaporã caracterizase por apresentar uma distribuição urbana menor que Bayeux ${ }^{10}$. Sugere-se, portanto, que pode haver diferenças no direcionamento das ações em saúde bucal, na oferta e no acesso aos serviços de saúde.

Quanto a proporção dos componentes do ceo-d/ $C P O-D$, verificou-se que $\circ$ cariado foi o principal aos 5 anos $(78,1 \%)$, assim como em João Pessoa $(77,8 \%)$, no Nordeste $(90,6 \%)^{2}$ e em Bayeux $(96,5 \%)^{16}$. Um estudo em Santa Rita do Pardo, Mato Grosso do Sul, também demonstrou maior número dentes cariados ${ }^{22}$. Observa-se que ainda há uma baixa oferta de serviços frente a maior demanda que necessita de tratamento restaurador. Os percentuais encontrados em João Pessoa foram melhores, possivelmente devido a maior facilidade de acesso aos serviços odontológicos na capital. Em contrapartida, a maior frequência no Nordeste pode ser decorrente do grande número de municípios de pequeno porte populacional, onde o acesso ao dentista é mais difícil e onde os programas de saúde são interrompidos com maior frequência ${ }^{22}$.

$\mathrm{Na}$ dentição permanente dos estudantes de Caaporã, o componente cariado também prevaleceu $(47,1 \%)$, intermediando o percentual de João Pessoa $(43,5 \%)$ e a média do Nordeste $(50,4 \%)^{2}$. Estes resultados divergem dos encontrados em Santa Rita do Pardo, Mato Grosso do Sul, no qual o percentual de dentes decíduos cariados $(41,2 \%)$ foi inferior ao da dentição permanente $(72,7 \%)^{22}$. Sugere-se que o maior acometimento de cárie nos dentes decíduos esteja associado à dependência que as crianças têm dos responsáveis para realizar a higienização bucal. Entretanto, muitos pais desconhecem a importância desta dentição para o bom desenvolvimento da dentição permanente, além de apresentarem hábitos alimentares inadequados, com dietas cariogênicas, que influenciam nas condições bucais das crianças ${ }^{4,5}$. Os resultados encontrados no município de Santa Rita do Prado/MS, que não recebe fluoretação artificial nas águas de consumo, podem ser justificados por se tratar de um município da zona rural, onde os hábitos alimentares e a dieta das crianças aos 5 anos pode ser menos cariogênica do que em cidades maiores, ou ainda devido a maioria da população 
de Santa Rita do Prado utilizar água de poço, visto que algumas localidades podem apresentar concentrações mais elevadas de fluoreto natural em águas subterrâneas quando comparadas a águas superficiais $^{23}$.

O CPO-D médio aos 12 anos em Caaporã $(2,44)$ foi inferior ao observado em Bayeux $(4,19)$ e na capital, João Pessoa (2,78), atingindo a meta proposta pela OMS para $\circ$ ano de 2000 nesta faixa etária (CPO-D $\leq 3)^{2,16}$. Todavia, os resultados encontrados na cidade de Campina Grande/ PB (prevalência de 70,5\% e CPO-D médio igual a 2,09$)$ foram inferiores aos dados de Caaporã, cuja prevalência foi de $79,4 \%$. Este fato pode ser decorrente do maior desenvolvimento social e econômico da cidade de Campina Grande ${ }^{20}$.

O acometimento de cárie aos 5 e 12 anos foi superior ao observado no município de Cascavel/PR, que apresentou, respectivamente, uma média de 2,42 e 1,91. Esta disparidade dos dados encontrados pode ser associada principalmente à fluoretação das águas de abastecimento público, assim como ao maior IDH $(0,78)$ do município de Cascavel ${ }^{24}$.

Estudo com crianças paulistas de 12 anos demonstrou uma diminuição acentuada do CPO-D de 3,72 para 1,37 entre 1998 e 2010. Dessa forma, ocorreu um aumento dos indivíduos livres de cárie de $20 \%$ para $53 \%$, sendo mais favorável que o resultado encontrado em Caaporã para a mesma faixa etária $(25,9 \%)^{1}$.

A média do CPO-D em paulistas de 15 aos 19 anos diminuiu de 6,44 para 3,29 , resultando no aumento do número de indivíduos livres de cárie de 9,6\% para $29,1 \%$. Pode-se afirmar que esses dados foram melhores que os encontrados no estudo de Caaporã (CPO-D=3,73 e 16,9\% sem experiência de cárie) devido ao maior acesso aos serviços odontológicos, à informação sobre higiene, à educação e à fluoretação das águas de abastecimento público, que são mais facilmente encontrados em municípios de maior porte ${ }^{14}$. Além disso, a prevalência de dentes perdidos por cárie nos escolares de 15 a 19 anos $(35 \%)$ foi superior ao esperado para o ano de $2000(\leq 15 \%)$ e $2010(n=0)^{10}$.

A prevalência das alterações periodontais em Caaporã foi de $28,6 \%$ e $40,7 \%$ para sangramento gengival e cálculo dentário, respectivamente. Segundo ○ SB Brasil 2010, 62,9\% das crianças brasileiras de 12 anos apresentaram todos os sextantes hígidos, enquanto no presente estudo, verificou-se que $67,8 \%$ das crianças de 12 anos e adolescentes de 15 a 19 anos apresentaram esta mesma condição em um percentual um pouco mais elevado que a média nacional e bem semelhante ao encontrado na região Sudeste $(67,9 \%)$, na qual este resultado foi considerado o mais satisfatório do Brasil $^{2}$.

A presença de cálculo aos 12 anos foi observada em 26,2\% das crianças residentes em Caaporã, resultado este próximo à média nacional $(23,7 \%)$. Os resultados do SB Brasil 2010 apontam que, ao analisar a prevalência das alterações periodontais, observa-se um aumento do sangramento gengival da infância para a adolescência ${ }^{2,22,24}$, e que aproximadamente $25 \%$ das crianças de 12 anos e $35 \%$ dos adolescentes de 15 a 19 anos apresentaram sangramento gengival ${ }^{2}$, estando em concordância com o presente estudo.

Em um estudo realizado no município de Patos, localizado no sertão da Paraíba, foi avaliado o nível de higiene oral de estudantes da Educação Infantil e do $1^{\circ}$ Ano do Ensino Fundamental, e observou-se que $62,1 \%$ apresentaram higiene regular, resultado próximo ao encontrado em Caaporã/PB (67,5\%). Todavia, foram realizadas atividades lúdicas, palestras e procedimentos restauradores simples por meio da técnica de ART (Tratamento Restaurador Atraumático) e observou-se melhoria na qualidade de higiene, com diminuição do percentual de escovação deficiente de $20,7 \%$ para $4,1 \%$, regular de $62,1 \%$ para $49,7 \%$, e aumento do índice de higienização boa, de $8,9 \%$ para $32 \%{ }^{25}$.

O desenvolvimento de atividades educativas e procedimentos restauradores simples contribuem para uma melhoria na qualidade da higiene, podendo diminuir cerca de $50 \%$ a prevalência de cárie $^{25}$. Esta medida pode ser planejada e realizada no município de Caaporã a fim de obter resultados mais concretos na redução da cárie. Sendo assim, as ações em saúde bucal devem ser direcionadas de acordo com a realidade encontrada no município a fim de atender as necessidades locais, melhorando as condições de saúde bucal da população e, consequentemente, alcançar as metas propostas pela OMS. 


\section{CONCLUSÃO}

A prevalência da cárie foi considerada alta aos 5 anos, baixa aos 12 e média dos 15 aos 19 anos. A higiene oral foi classificada como regular, sendo mais deficiente nas crianças de 5 anos. Não houve associação da cárie com sexo e higiene.

Os resultados deste estudo tornam possível o direcionamento das decisões em saúde pública, visto que, como não havia dados epidemiológicos coletados anteriormente em Caaporã, os resultados obtidos no presente estudo apresentam a realidade epidemiológica do município e contribuem com informações sobre a prevalência e gravidade de cárie dentária, visando auxiliar na ampliação da cobertura de saúde bucal e no desenvolvimento de ações adequadas de promoção de saúde.

\section{AGRADECIMENTOS}

Ao Conselho Nacional de Desenvolvimento Científico e Tecnológico (CNPq) pelo financiamento do presente estudo - Processo 403343/2012-7.

\section{REFERÊNCIAS}

1. Sousa MLR, Rando-Meirelles MPM, Tôrres LHN, Frias AC. Cárie dentária e necessidades de tratamento em adolescentes paulistas. Rev. Saúde Pública. 2013;47(Suppl3):50-58. Doi: Cárie dentária e necessidades de tratamento em adolescentes paulistas. Rev. Saúde Pública. doi: 10.1590/S0034-8910.2013047004340

2. Brasil. Ministério da Saúde. Pesquisa Nacional de Saúde Bucal - 2010: Resultados principais. Brasília. DF. 2011.

3. Rihs LB, Sousa MLR, Cypriano S, Abdalla NM, Guidini DDN, Amgarten, C. Atividade de cárie na dentição decídua, Indaiatuba. Caderno de Saúde
Pública. 2007;23(3):593-600. doi: 10.1590/ s0102-311X2007000300018

4. Li Y, Wang W. Predicting Caries in Permanent Teeth from Caries in Primary Teeth: An Eight-year Cohort Study. Journal of Dental Research. 2002;81(8):561-66. doi: $10.1177 / 154405910208100812$

5. Eleutério ASL, Cota ALS, Kobayashi TY, Silva SMB. Avaliação Clínica da Saúde Bucal de Crianças dos Municípios de Alfenas e Areado, Minas Gerais, Brasil. Pesq. Bras. Odontoped. Clin. Integr. 2012;1 2(2):195-201. doi: 10.4034/ PBOCl.2012.122.07

6. Moraes ES, Valença AMG. Prevalência de gengivite e periodontite em crianças de 3 a 5 anos na cidade de Aracajú (SE). Cienc Odontol Bras. 2003;6(4):87-94.

7. Brasil. Ministério da Saúde. Programa Saúde na Escola [Internet]. 2016 [cited $2016 \mathrm{Fev}$ 29]. Available from: http://dab.saude.gov.br/ portaldab/pse.php

8. Lakatos EM, Marconi MA. Fundamentos de metodologia científica. $6^{a}$ ed. São Paulo: Atlas; 2007.

9. World Health Organization - WHO. Oral health surveys: basic methods. 4th ed. Geneva: World Health Organization; 1997.

10. IBGE. Instituto Brasileiro de Geografia e Estatística [Internet]. 2014 [cited 2014 Jun 28]. Available from: http://www.ibge.gov.br

11. Prefeitura Municipal de Caaporã-PB [Internet]. 2014 [cited 2014 Jun 28]. Available from: http:// www.caapora.pb.gov.br/a-cidade/

12. Saúde Bucal Coletiva. Adaptado por Frias AC, Junqueira SR [Internet]. 2016 [cited 2016 Fev 02]. Available from: http://www.fo.usp.br/wp-content/ uploads/GTextoSBC

13. Gomes VE, Silva DD. A importância do controle de placa dental na clínica odontológica. Arquivos em Odontologia. 2010;46(1):22-27 
14. Musso VF, Zandonade E, Emmerich AO.

Desigualdades na distribuição da cárie dentária aos 12 anos no Brasil. Rev. Bras. Pesq. Saúde. 2013;15(2):64-72. doi: 10.21722/rbps.v0i0.5676

\section{Freire $M C M$, Reis SCGB, Figueiredo N, Peres}

KG, Moreira RS, Antunes JLF. Determinantes individuais e contextuais da cárie em crianças brasileiras de 12 anos em 2010. Rev. Saúde Pública. 2013;47(Supp3):40-49. doi: 10.1590/ S0034-8910.2013047004322

16. Azevedo AC, Valença AMG, Lima Neto EA. Perfil epidemiológico da cárie dentária em escolares de 5 e 12 anos residentes no Município de Bayeux, Paraíba. Arq. Odontol. 2012; 48(2):68-75. doi: 10.7308/ aodontol/2012.48.2.02

17. Amaral RC, Batista MJ, Cypriano S, Sousa MLR. Cuidados odontológicos e fatores associados em escolares do município de Indaiatuba-SP. Arq. Odontol. 2013;49(1):39-44. doi: 10.7308/ aodontol/2013.49.1.06

18. Gradella CMF, Oliveira LB, Ardenghi TM, Bonecker M. Epidemiologia da cárie dentária em crianças de 5 a 59 meses de idade no município de Macapá. AP. RGO. 2007;55(4):329-34.

19. Eskenazi EMS, Sousa KG, Agostini LTP, Barbosa TS, Castelo PM. Avaliação da experiência de cárie e qualidade de vida relacionada à saúde bucal de escolares. Rev. Bras. Promoç. Saúde. 2015; 28(2):198-205. doi: 10.5020/18061230.2015. p 198

20. Moura C, Cavalcanti AL, Bezerra PKM. Prevalência de cárie dentária em escolares de 12 anos de idade, Campina Grande, Paraíba, Brasil: enfoque socioeconômico. Rev. odonto ciênc. 2008;23(3):256-62.

21. Santos NCN, Alves TDB, Freitas VS, Jamelli SR, Sarinho ESC. A saúde bucal de adolescentes: aspectos de higiene, de cárie dentária e doença periodontal nas cidades de Recife, Pernambuco e Feira de Santana, Bahia. Rev. Ciência \& Saúde Coletiva. 2007;1 2(5):1 155-66. doi: 10.1590/ S1413-81232007000500012
22. Pontes ERJC, Gimenes ACR. Prevalência de cárie dentária e condições periodontais de escolares de 5 a 12 anos de idade, em um município rural brasileiro. RGO - Rev Gaucha Odontol. 2011 ;59(4):577-82

23. Alves JC. Determinação de íons fluoreto em águas, por titulação condutimétrica com nitrato de Lantânio [Dissertação]. Universidade de São Paulo; 1986.

24. Berti M, Furlanetto DLC, Walker MMS, Baltazar $M M M$, Bianchi FJ. Levantamento epidemiológico de cárie dentária em escolares de 5 e 12 anos de idade do município de Cascavel, PR. Rev. Caderno de Saúde Coletiva. 2013;21 (4):403-6. doi: $10.1590 / \mathrm{S} 1414-462 \times 2013000400007$

25. Carvalho THL, Pinheiro NMS, Santos JMA, Costa LED, Queiroz FS, Nóbrega CBC. Estratégias de promoção de saúde para crianças em idade pré-escolar do município de Patos-PB. Rev. odontol. UNESP. 2013;42(6):426-31. doi: 10.1590/S180725772013000600006 